\title{
Sarcoma cardíaco metastático a miembros inferiores
}

\author{
Yelson A Picón-Jaimes', Javier E Orozco-Chinome², Leec FD Ortega-Uribe ${ }^{3}$
}

\section{RESUMEN}

Introducción. Las neoplasias de corazón son patologías infrecuentes con tasas de incidencias en reportes de autopsias menores al $0.02 \%$. El $75 \%$ de esos tumores son de comportamiento benigno y solo el $25 \%$ restante se considera cáncer. Aunque se presentan en ambos sexos, la relación hombres mujeres es de 2.5:1 respectivamente. Presentación del caso. Reportamos el caso de un paciente de 41 años a quien se diagnosticó con un sarcoma primario de corazón, en atrio izquierdo, con inmunohistoquímica compatible con sarcoma indiferenciado con metástasis a miembros inferiores, que debutó con deterioro de la clase funcional y pérdida de peso involuntaria, lo cual lo llevó a consultar al médico.

Discusión. Los sarcomas indiferenciados constituyen una patología poco frecuente en la literatura médica, encontrando solo unos pocos reportes de casos que comparten datos relacionados con el sitio de localización de la lesión y los síntomas clínicos de los pacientes. Sin embargo, aquí se pone de manifiesto un extraño caso de metástasis a miembros inferiores, el cual fue considerado luego de descartar la presencia de un foramen oval permeable o lesiones pulmonares que hicieran pensar en una secuencia de migración tumoral diferente.

Conclusiones. Se trata de una patología compleja con pobre pronóstico a largo plazo, la cual requiere mayor investigación y tratamiento multifactorial con equipos multidisciplinarios para mejorar la calidad de vida de los pacientes.

Palabras clave: sarcoma; neoplasias del mediastino; neoplasias; insuficiencia cardíaca; cirugía torácica.

\footnotetext{
${ }^{1}$ Universidad Pedro de Valdivia, Santiago, Chile.

${ }^{2}$ Red Salud, Santiago, Chile.

${ }^{3}$ Clínica Chicamocha, Bucaramanga, Colombia.
}

Correspondencia: Yelson Alejandro Picón Jaimes. Dirección: Av Pocuro 2260, Providencia, Región Metropolitana, Santiago, Chile. Teléfono: +56 948991158 .

Correo electrónico: ypicon@unab.edu.co 


\title{
Cardiac sarcoma with metastasis to the lower limbs
}

\begin{abstract}
Introduction. Neoplasms of the heart are infrequent pathologies with incidence rates in autopsy reports less than $0.02 \% .75 \%$ of these tumors are benign and only the remaining $25 \%$ are considered cancerous. Although they occur in both sexes, the male to female ratio is $2.5: 1$ respectively.

Case report. We report the case of a 41 -year-old patient who was diagnosed with a primary heart sarcoma, in the left atrium, with immunohistochemistry compatible with undifferentiated sarcoma with metastases to the lower limbs, who debuted with impairment of functional class and loss of involuntary weight which led him to consult the doctor.

Discussion. Undifferentiated sarcomas constitute a rare pathology in the medical literature, finding only a few cases reports that share data, such as the location of the lesion and the clinical symptoms of the patients. However, this case reveals a strange case of metastasis to lower limbs, which was considered, after ruling out the presence of a patent foramen ovale or lung lesions that suggested a different tumor migration sequence.

Conclusions. This is a complex pathology with a poor long-term prognosis that requires more research and multifactorial treatment with multidisciplinary teams to improve the quality of life of patients
\end{abstract}

Key words: sarcoma; mediastinal neoplasms; neoplasms; heart failure; thoracic surgery. 


\title{
Sarcoma cardíaco metastático dos membros inferiores
}

\begin{abstract}
RESUMO
Introdução. As neoplasias do coração são patologias pouco frequentes, com taxas de incidência em relatórios de autópsia inferiores a 0,02\%. 75\% desses tumores são benignos e apenas $25 \%$ restantes são considerados câncer. Embora ocorram em ambos os sexos, a proporção entre homens e mulheres é de 2,5: 1 respectivamente.

Apresentação do caso. Relatamos o caso de um paciente de 41 anos de idade com diagnóstico de sarcoma primário do coração, no átrio esquerdo, com imuno-histoquímica compatível com sarcoma indiferenciado com metástase nos membros inferiores, que começou com deterioração da classe funcional e perda involuntária de peso, o que o levou a consultar o médico.

Discussão. Os sarcomas indiferenciados constituem uma patologia rara na literatura médica, encontrando apenas alguns relatos de casos que compartilham dados relacionados ao local da localização da lesão e aos sintomas clínicos dos pacientes. No entanto, aqui é revelado um caso estranho de metástase nos membros inferiores, que foi considerado após descartar a presença de forame oval permeável ou lesões pulmonares que sugerem uma sequência de migração tumoral diferente.
\end{abstract}

Conclusões. É uma patologia complexa, com mau prognóstico a longo prazo, que requer mais investigação e tratamento multifatorial com equipes multidisciplinares para melhorar a qualidade de vida dos pacientes.

Palavras-chave: sarcoma; neoplasias do mediastino; neoplasias; insuficiência cardíaca; cirurgia toráxica. 


\section{INTRODUCCIÓN}

Las neoplasias de corazón son entidades extremadamente raras con incidencias reportadas en series de necropsias del $0.02 \%$; de estas, solo entre el $15 \%$ y el $20 \%$ son de comportamiento maligno, lo que generalmente obedece a un tumor de tipo sarcoma (1).

Los sarcomas cardiacos se originan de células pluripotenciales mesenquimales y poseen un amplio rango de diferenciación celular por lo cual sus bases moleculares no son del todo claras (2). Dichos sarcomas ocurren tanto en hombres como en mujeres $y$, en términos generales, pueden desarrollarse en cualquier localización dentro del corazón, siendo un sitio frecuente de aparición el atrio izquierdo; sin embargo, cuando se localizan en el atrio derecho debe considerarse, como primera opción, que representen un proceso metastásico, excepto por el angiosarcoma el cual preferentemente se desarrolla en este sitio $(2,3)$.

Usualmente, estas patologías permanecen asintomáticas hasta que logran un tamaño considerable que, por su localización en atrio izquierdo, desencadenan manifestaciones clínicas como la disnea con obstrucción al flujo hacia ventrículo izquierdo, por obstrucción de las venas pulmonares y congestión retrograda que produce hipertensión arterial pulmonar. En cuanto a las metástasis, lo usual es que esté a distancia, especialmente en pulmón, riñón, hígado, glándulas suprarrenales y hueso $(2,4)$.

El objetivo de este manuscrito es presentar un caso de una patología tumoral poco frecuente que, adicionalmente, se acompañó de otra lesión tumoral en miembro inferior, por lo cual, y debido a los antecedentes del paciente, se trataba de un tumor cardiaco maligno metastásico; lo anterior, hace de este un caso más infrecuente por el sitio de localización de la segunda neoplasia.

\section{PRESENTACIÓN DEL CASO}

Reportamos el caso de un paciente masculino de 41 años, sin antecedentes médicos de importancia, quien decide asistir para atención médica por disnea de pequeños esfuerzos, de dos meses de evolución, y pérdida progresiva e involuntaria de aproximadamente ocho kilogramos.

El paciente manifestaba que algunas actividades sencillas, como el subir las escaleras de su casa, le desencadenaban sensación de cansancio extremo que no guardaba relación con el nivel de actividad física realizado, teniendo en cuenta que él era una persona deportista con hábitos nutricionales vegetarianos y peso saludable. Había asistido ambulatoriamente a consulta médica con múltiples especialistas, incluyendo gastroenterología, donde se determinó que los síntomas no eran explicados por reflujo gastroesofágico, 
ya que se trataban de un franco deterioro de la clase funcional, motivo por el cual el paciente fue remitido al servicio de urgencias. A su ingreso, el examen físico inicial evidenciaba temperatura de $36.8^{\circ} \mathrm{C}$, presión arterial en brazo izquierdo de $145 / 82 \mathrm{mmHg}$, oxigenometría de pulso del $95 \%$, frecuencia cardiaca en 87 latidos por minuto.

En el examen físico segmentario se identificó ingurgitación yugular, ruidos cardiacos rítmicos de baja intensidad, soplo sistólico, regurgitante grado II/VI, de predominio en foco mitral y crepitancias en bases pulmonares. La tomografía contrastada reveló lesión ocupante de espacio en el atrio izquierdo que se extendía hasta la válvula mitral (Figura 1); por otra parte, la ultrasonografía cardiaca evidenció lesión tumoral que ocupaba el $95 \%$ del atrio izquierdo, adherida al techo atrial, comprometiendo la llegada de las venas pulmonares izquierdas superior e inferior, presión pulmonar sistólica de $100 \mathrm{mmHg}$, aplanamiento del septum interventricular y fracción de eyección del ventrículo izquierdo (FEVI) de $60 \%$. Adicionalmente, el paciente manifestó sensación de masa en muslo derecho por lo cual se realizó ecografía encontrando masa hipoecoica, de aspecto sólido de 78 x 47x 97 mm, definida en el plano muscular, en la cara posteroexterna del muslo adyacente a la diáfisis proximal femoral. El usuario informó que en el 2015 le había sido extraída una masa de miembro inferior izquierdo que correspondía a un tumor fusocelular sarcomatoide. Se decidió hospitalizar en unidad de cuidados intensivos por riesgo de muerte súbita y se iniciaron trámites de traslado a institución de mayor complejidad.

Figura 1 Tomografía contrastada que evidencia masa en aurícula izquierda

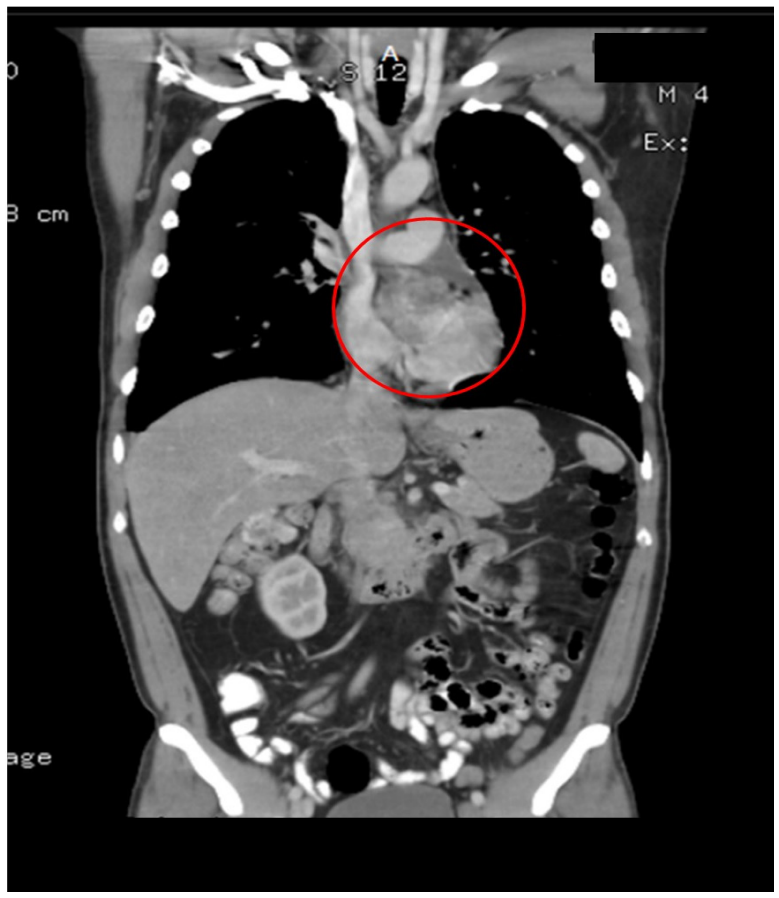

En la nueva institución fue valorado por el departamento de cirugía cardiovascular, donde le realizaron estudios de extensión, en búsqueda de metástasis, y exámenes de laboratorio adicionales, gracias a lo cual se encontró alteración de la creatincinasa total, la proteína $C$ reativa y la velocidad de sedimentación globular (Tabla 1). 
Tabla 1 Exámenes de laboratorio realizados al paciente durante su ingreso al servicio de urgencias

\begin{tabular}{cccc}
\hline EXAMEN & RESULTADO & EXAMEN & RESULTADO \\
\hline Creatinina & $0.90 \mathrm{mg} / \mathrm{dL}$ & C3 fracción complemento & $1.79 \mathrm{~g} / \mathrm{L}$ \\
\hline Deshidrogenasa láctica & $179 \mathrm{U} / \mathrm{L}$ & C4 fracción complemento & $0.28 \mathrm{~g} / \mathrm{L}$ \\
\hline Fosfatasa alcalina & $70 \mathrm{U} / \mathrm{I}$ & Anticuerpos anti LA/SSB & $0.90 \mathrm{U} / \mathrm{mL}$ \\
\hline Gama glutamil transferasa & $16.3 \mathrm{U} / \mathrm{I}$ & Anticuerpos anti RNP & $0.30 \mathrm{U} / \mathrm{mL}$ \\
\hline Alanino aminotransferasa & $20.3 \mathrm{U} / \mathrm{I}$ & Anticuerpos anti-RO/SSA & $1.40 \mathrm{U} / \mathrm{mL}$ \\
\hline Aspartato aminotransferasa & $13.9 \mathrm{U} / \mathrm{I}$ & Anticuerpos anti-SM & $1.30 \mathrm{U} / \mathrm{mL}$ \\
\hline Anticuerpos DNA & Negativo & Anticuerpos C-ANCA y PANCA & Negativo \\
\hline Anticuerpos anti-músculo liso & Negativo & Hemoglobina & $13.0 \mathrm{~g} / \mathrm{dL}$ \\
\hline CPK & $209 \mathrm{U} / \mathrm{I}{ }^{* *}$ & Leucocitos & $8.030 \mathrm{~mm}{ }^{3}$ \\
\hline Glicemia & $91.95 \mathrm{mg} / \mathrm{dL}$ & Recuento plaquetario & $342.000 \mathrm{~mm}$ \\
\hline Proteína C reactiva & $56.81 \mathrm{mg} / \mathrm{L} * *$ & Velocidad de sedimentación & $34.0 \mathrm{~mm} / \mathrm{h}^{* *}$ \\
\hline Hormona tiro-estimulante & $2.37 \mathrm{uUI} / \mathrm{mL}$ & Vitamina B12 & $280 \mathrm{pg} / \mathrm{mL}$ \\
\hline
\end{tabular}

Días después, el paciente fue llevado al quirófano donde, previa anestesia general, le practicaron esternotomía mediana y exposición intrapericárdica, resecando masa completa (Figura 2), con preservación de la válvula mitral. Se comprobó funcionalidad de la válvula mitral con ecocardiograma transesofágico, procediendo con el cierre por planos y enviando al paciente a recuperación, sin complicaciones luego de cuatro horas y 30 minutos de procedimiento.

En unidad de cardiointensivismo, se realizó ecocardiograma transtorácico posquirúrgico que reportó ventrículo izquierdo de dimensiones conservadas, hipocinesia leve asincrónica septal, FEVI del $60 \%$, atrio izquierda sin visualización de material neoplásico en su interior, insuficiencia mitral II/IV y derrame pericárdico leve. El paciente continuó con tendencia a la mejoría y, luego de terminar plan de rehabilitación cardiopulmonar, fue dado de alta.

El reporte de patología de la lesión extirpada reveló a la microscopía lesión tumoral, constituida por células grandes con marcada atipia citológica, tipo osteoclasto like con hasta 3 mitosis por campo; componente inflamatorio constituido por plasmocitos, linfocitos reactivos alrededor de 
Figura 2 Masa resecada de aurícula izquierda

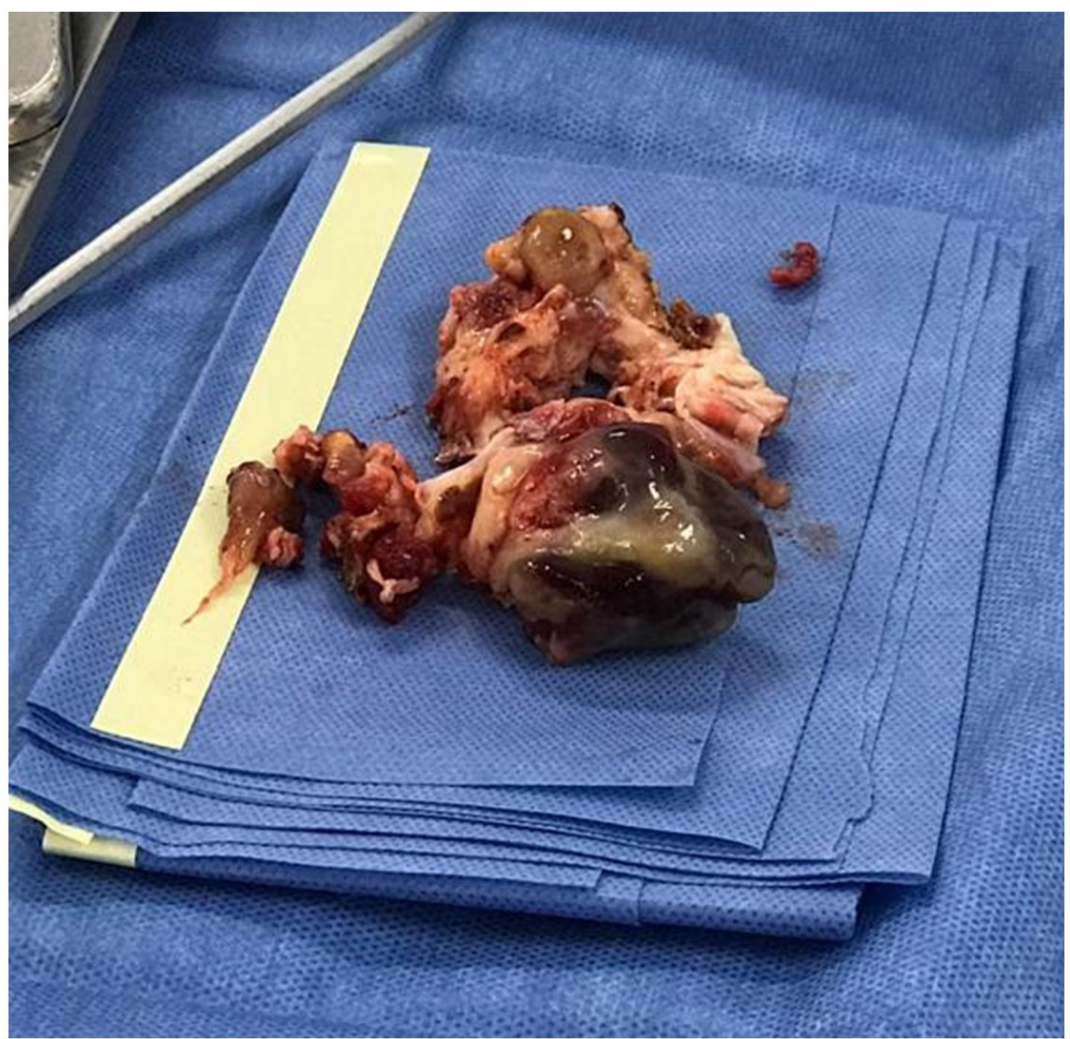

los vasos, con extensas áreas de necrosis tumoral. Índice de proliferación KI 67 del 40 \%. Por hallazgos de inmunohistoquímica se determinó lesión compatible con sarcoma de alto grado indiferenciado, sin compromiso linfovascular.

En un tiempo quirúrgico posterior, se realizó resección de masa de muslo derecho (Figura 3), la cual fue enviada a patología, desde donde se reportó sarcoma pleomórfico de alto grado, extensamente necrosado sin invasión linfovascular. En la actualidad, el paciente vive, con una buena clase funcional, en manejo concomitante por oncología para controles periódicos de la enfermedad metastásica. 
Figura 3 Masa resecada de miembro inferior derecho

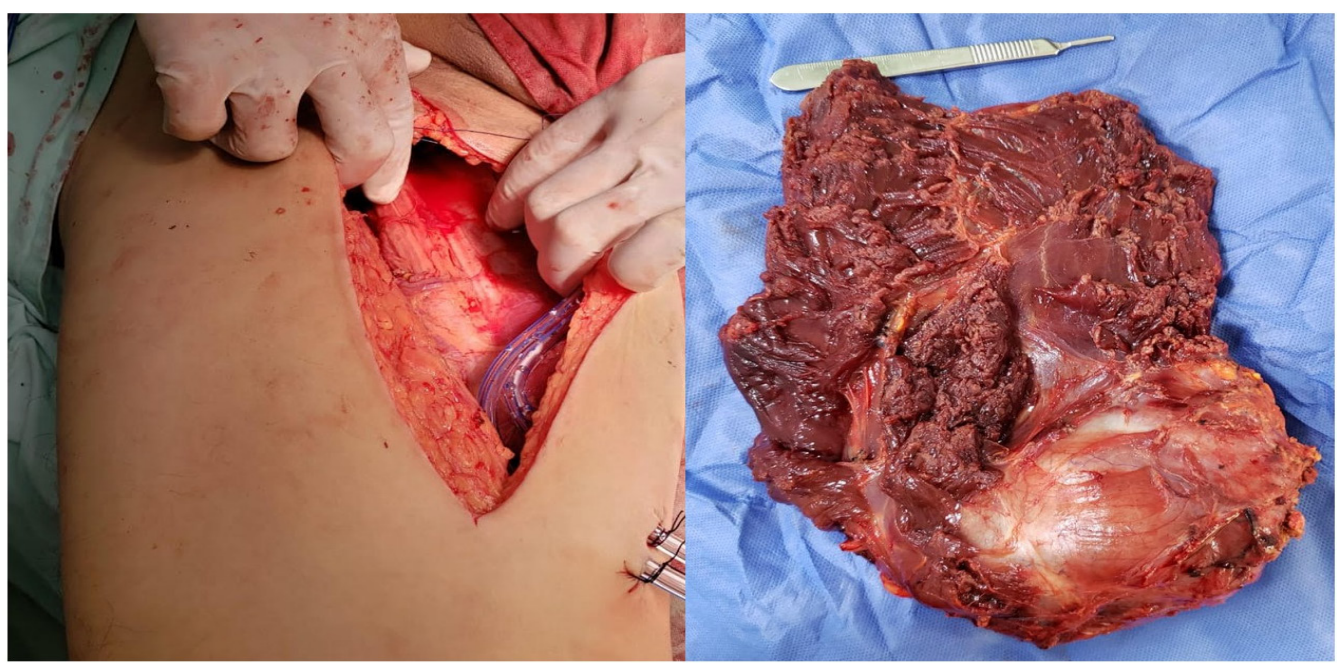

\section{DISCUSIÓN}

Se llevó a cabo una búsqueda en diferentes bases de datos, sin encontrar un caso similar con metástasis a miembros inferiores. Los sarcomas cardiacos suelen aparecer entre la tercera y quinta década de vida, como es el caso de este paciente; asimismo, se considera que su incidencia se subestima debido a que usualmente se reportan durante autopsias, ya que tienden a comprometer de forma abrupta la vida, presentándose con muerte súbita como consecuencia del daño cardiopulmonar ocasionado por el efecto de masa y obstrucción al flujo sanguíneo (1). Según la organización mundial de la salud, la distribución de los sarcomas de corazón por orden de frecuencia es: en primer lugar, el angiosarcoma con el $37 \%$ de los casos, seguido del sarcoma indiferenciado que se identifica hasta en el $24 \%$ de las veces, el histiocitoma maligno fibroso del $11 \%$ al $24 \%$ y el porcentaje restante está dividido entre el leiomiosarcoma, el rabdomiosarcoma, fibrosarcoma y osteosarcoma (2).

El sarcoma indiferenciado, que para algunos autores comprende también al sarcoma pleomórfico y al histiocitoma fibroso maligno, se localiza generalmente en el atrio izquierdo con extensión a la válvula mitral, causando obstrucción del orificio valvular y afectación venocapilar pulmonar con hipertensión en estos vasos sanguíneos (5). Puede verse como una masa polipoide firme, de 
color blanco nacarado con áreas de necrosis o hemorragia, además, a la histología se identifica un sarcoma de alto grado, con celularidad variable, pleomorfismo y alta tasa de mitosis (6).

El diagnóstico de esta patología es netamente histológico, sin embargo, el abordaje inicial se realiza con las imágenes diagnósticas, dentro de las cuales el ecocardiograma transtorácico es una excelente herramienta inicial que permite la identificación de la masa tumoral. Otras imágenes que ayudan a orientar mejor la localización del tumor son la ecocardiografía transesofágica, la tomografía contrastada y la resonancia magnética, las cuales ayudan a desarrollar el plan quirúrgico en estos pacientes $(7,8)$.

El tratamiento consiste en la resección quirúrgica de la lesión primaria y de las lesiones metastásicas; en algunos casos, se precisa de la ayuda con quimioterapia y radiación con fines paliativos, pues se trata de tumores de alto grado de malignidad con una alta frecuencia de recurrencia o metástasis a órganos como pulmón y cerebro principalmente (9). El pronóstico de estos pacientes suele ser malo, con sobrevida a 36 meses menor del $20 \%$; incluso, hay autores que afirman que en la actualidad no hay un centro con la suficiente experiencia en este tipo de tumores que permita una mejor evaluación de la sobrevida de estos individuos $(3,10)$.
Los sarcomas metastásicos a corazón presentan, usualmente, lesiones en las cámaras derechas y se asocian a la vía de diseminación hematógena, como lo reporta en su caso Rivera et. al., cuando describe un sarcoma sinovial de pie con metástasis a ventrículo derecho (11). Sin embargo, en este caso el tumor de corazón se encontraba en el atrio izquierdo, sitio que coincide con el de aparición de los sarcomas primarios indiferenciados de corazón; adicionalmente, en los ecocardiogramas no se evidenciaba permanencia o permeabilidad del foramen oval interauricular, por lo cual se consideró una neoplasia primaria de corazón con metástasis por vía hematógena con siembras tumorales en ambos miembros inferiores; por esta razón, este caso no es comparable con otros en la literatura médica actual. Es imprescindible la presentación de este caso para invitar a investigadores a considerar los miembros inferiores como un sitio de metástasis de los sarcomas primarios de corazón, así como abrir el campo para dilucidar el mecanismo por el cual se dan este tipo de complicaciones.

\section{CONCLUSIONES}

Los sarcomas indiferenciados de corazón son una forma rara de sarcoma, estos ocurren esporádicamente y en baja proporción en la población general. Estos tumores son agresivos y se tratan de forma ideal en un centro de cáncer con experiencia, con un enfoque multidisciplinario. EI 
pronóstico se asocia con el tamaño de la lesión, márgenes libres en el momento de la primera cirugía y presencia o no de metástasis. En este caso en particular, la lesión tumoral presentaba metástasis a miembro inferior, lo cual no fue posible contrastar con la literatura médica actual, lo que permitió considerar como posible vía de diseminación la hematógena, considerando que los ganglios circundantes se encontraban libres de tumor.

Las nuevas opciones de tratamiento van encaminadas a la detección precoz y determinación de terapias adyuvantes como la quimioterapia y la radiación.

\section{AGRADECIMIENTOS}

A la institución de salud por permitir el acceso a la información y al paciente quien de manera voluntaria colaboró con la entrevista y dio su consentimiento informado para la publicación de la historia de su patología, respetando su autonomía y protegiendo sus datos personales.

\section{CONFLICTOS DE INTERÉS}

Los autores declaran no tener conflictos de interés.

\section{FINANCIACIÓN}

No se contó con financiación por parte de entidades externas.

\section{REFERENCIAS}

1. Díaz-García J, Carrera-Patiño F, Aquino-Matus J, Tenorio E, Martínez H. Sarcoma indiferenciado primario de corazón. A propósito de un caso. Rev Española Casos Clínicos en Med Interna. 2019;4(1):6-9. https://doi.org/10.32818/ RECCMI.A4N1A3

2. Bosson C, Mota J. Tumores primarios cardíacos y pericárdicos (Parte III): tumores cardíacos malignos. Av Cardiol. 2016;36(3):151-68.

3. Ogechukwu C, Christopher N, Christoph S, Etinosasere $\mathrm{O}$, Jose $\mathrm{B}$. The Insidious Cardiac Tumor: A Primary Left Atrium Intimal Cardiac Sarcoma in a Young Patient. Case Reports Cardiol. 2019;2019:4-7. https://doi. org/10.1155/2019/7245676

4. Vaitiekiene A, Vaitiekus D, Urbonaite L, Jankauskas A, Portacenko J, Lapinskas T, et al. Multidisciplinary approach to rare primary cardiac sarcoma: a case report and review. BMC Cancer. 2019;19(529):2-7. https://doi. org/10.1186/s12885-019-5705-2

5. Chaves A, Alvarado E, Dam C, Castillo O, Induni $E$, Méndez $E$, et al. Sarcoma Primario del Pericardio Reporte de Caso. Rev Cl EMed UCR. 2018;8(I):1-6. 
6. Fu X, Niu W, Li J, Kiliti AJ, Al-ahmadie HA, Gao SP, et al. Activating mutation of PDGFRB gene in a rare cardiac undifferentiated intimal sarcoma of the left atrium: a case report. 2017;8(46):81709-16. https://doi. org/10.18632/oncotarget.20700

7. Rodríguez $A$, Flores $A$, Estévez $N$, Bencomo $\mathrm{L}$, Álvarez R, Zorio B. Tumor cardiaco primario maligno. A propósito de un caso. Rev Cuba Cardiología y Cirugía Cardiovasc. 2016;22(2):7-9.

8. Raphael K, Martínez A, Clements S, Isiadinso I. Role of Multimodal Cardiac Imaging. Texas Hear Inst J. 2019;46(1):28-31. https://doi. org/10.14503/THIJ-16-5896

9. Yufan W, Lynn M, Evertt M, Gregory S, Mark B, Kristen $\mathrm{G}$. The impact of postoperative therapy on primary cardiac sarcoma. JThorac Cardiovasc Surg [Internet]. 2018;156(6):2194-203. https://doi.org/10.1016/j.jtcvs.2018.04.127

10. Murinello A. Angiosarcoma cardíaco: una neoplasia rara y de difícil diagnóstico Cardiac angiosarcoma: a rare neoplasia of difficult diagnosis. Salud(i)Ciencia 2014;20:394-8.

11. Rivera $M$, Chagolla $M$, Vargas $A$, López A, Martínez K. Sarcoma sinovial metastásico del ventrículo derecho. Reporte de un caso y revisión de la bibliografía. Evid Med invest salud. 2016;9:179-82. 